\title{
A Novel Hybrid Approach for Medical Image Compression
}

\author{
Sajid Ullah Khan ${ }^{1,}$, Najeeb Ullah ${ }^{2}$, Imran Ahmed ${ }^{3}$, Wang Yin Chai ${ }^{1}$ and Shahid Ullah ${ }^{1}$ \\ ${ }^{I}$ FCSIT, Universiti Malaysia Sarawak, Malaysia; ${ }^{2}$ CECOS University of IT and Emerging Sciences, KPK, Peshawar, \\ Pakistan; ${ }^{3}$ Institute of Management Sciences, Peshawar, Pakistan
}

\begin{abstract}
Background: The area of image processing extends from basic image compression model to high end applications such as astronomical data processing and medical image processing. Images have vast features and coding them into lower bit rates results in the loss of information.

Method: This study proposes a novel hybrid approach to compress the image no loss of information and to maintain reduced processing overhead.

Disscusion: To validate the proposed approach, the suggested method is tested on different medical samples and the obtained results are compared with results retrived through the conventional JPEG based coding approach.
\end{abstract}

\begin{tabular}{l} 
A R T I C L E H I S T O R Y \\
\hline Received: February 07, 2017 \\
Revised: March 22, 2017 \\
Accepted: April 02, 2017 \\
DOI: \\
10.2174/1573405613666170428162650
\end{tabular}

Conclusion: The experimental results show that the proposed approach performs efficiently and can be implemented in realworld applications.

Keywords: Image compression, coefficients learning, learning based coding, probabilistic decision algorithm, hybrid approach.

\section{INTRODUCTION}

Medical image compression is a mandatory process to transmit multiple images with lower bit rate and also needs to achieve a good image quality at the receiver side in image processing applications like progressive image encoding, multimedia transmitting, image browsing, etc. The major objective of the image compression is to compress the image while preserving useful details based on given requirements. Along with this, encoding in the noise environment becomes too complex and results in the heavier degradation in the quality of the image. In various applications of image processing, images are processed at the capturing point, and communicated over a channel to a monitoring or storage location. The advancement of image processing, has given the advantage of automation in various image coding applications, among which medical image processing is one of the prime areas. Medical diagnosis has always remained a time taking and sensitive approach for medical diagnosis. Previously, several approaches have been proposed for compressing and encoding of images. According to the rise of telemedicine and the use of digital medical images, the fast and efficient coding algorithms are needed for the medical field [1]. A comparison of Curvelet with Wavelet based compression was made for standard images like Lena, and Barbara have presented [2]. This technique is very useful for natural images. Multi-Resolution Analysis approach was introduced to compress medical images [3]. However, it used wavelet based compression that can compress image

*Address correspondence to this author at the FCSIT, Universiti Malaysia Sarawak, Malaysia; Tel: 0060149154360; E-mail: khansajidmalik@gmail.com smoothly but increases computational cost. A recent Wavelet Based volumetric medical image compression technique was introduced to compress medical images [4]. This technique fails when the image size increases and cannot reduce processing overhead and processing bandwidth. A wavelet decomposition followed by the correlation analysis of coefficients was presented to achieve high quality compression rate [5] but it cannot retain the accuracy. JPEG2000 employed the 2-D discrete wavelet transform (DWT) [6]. It decomposes input into four spatial frequency sub bands and makes smaller code blocks. However, smaller code-block sizes result in increased decoding times due to increased fetches and the increased overhead of decoding smaller code-blocks. The idea can reduce the entropy of transformed images and can increase compression [7]. But it only works well for lossless compression of medical images. The compression of medical images using wavelet transform was introduced [8]. However, it selects the unwanted coefficients from the sub-bands as well which leads to unwanted information. A compression using Integer Transforms and Predictive coding technique was introduced [9] but it also selects the non-relevant coefficients that degrade image quality. A technique EWISTARS was proposed [16] but unwanted coefficients were selected which increase further processing. An efficient technique for Hyper Spectral Image HSI compression was introduced based on (DWT) and Tucker Decomposition (TD) with Adaptive Least Squares (ALS) method [15]. This technique exploits both the spatial and spectral information in the images. However, the issue of image coefficients selection and processing with retaining the required level of accuracy is a major problem observed in this approach. 